\title{
THE CONTRIBUTION OF THE GALACTIC BULGE TO THE
}

\section{GALACTIC ROTATION CURVE}

\author{
I.V. PETROVSKAYA \\ Astronomical Institute of St. Petersburg University, Bibliotechnaya pl. D, St. Petersburg, \\ 198904, Russia \\ and \\ S. NINKOVIĆ \\ Astronomical Observatory, Volgina 7, 11050 Belgrade, Yugoslavia
}

It is not always clear what the bulge of the Galaxy is: a region close to the centre, a subsystem formed by a distinct population, or a mixture of populations but characterised by its own mass distribution. We consider the bulge of the Milky Way as a subsystem and thus contributing to the galactic gravitation field. We want to estimate the contribution of the galactic bulge to the rotation curve.

The rotation curve was obtained from the HII data and HI data (tangential points, $21 \mathrm{~cm}$ line profile). We also added the HI results by Merrifield obtained from the hydrogen layer thickness. The distances to HII regions are known in kiloparsecs whereas the distances to the HI clouds are obtained as a ratio to $R_{0}$ - the distance of the sun from the galactic centre. The best agreement of HII and HI data was found for $R_{0}=7.9 \mathrm{kpc}$ (Nikiforov, I.I. \&. Petrovskaya, I.V., in prep.) and we adopted $208.6 \mathrm{~km} / \mathrm{s}$ for the linear velocity of the LSR (Kerr, F.J. and Lynden-Bell, D.,(1986, Mon.Not.R.Astron.Soc., 221, 1023) recommend $26.4 \mathrm{~km} / \mathrm{s} / \mathrm{kpc}$ as the value of the LSR angular velocity ).

In order to explain the circular velocity of the Galaxy we propose a model containing four subsystems: 1) the core; 2) the bulge; 3) the disk; 4) the corona. We try to explain some feature of the rotation curve, namely, the sharp maximum near the galactic centre followed by a corresponding minimum somewhat father from the centre (core) and the flat part of the curve at the larger distances (corona). For the core we assume a model of homogeneous spheroid. Its semiaxis major is equal to the distance at which the maximum occurs, the axial ratio is between 0.4 and 1 depending on the fit to the rotation curve. For the disk we adopted the truncated exponential model (Casertano, S., 1983, Mon.Not.R.Astron.Soc., 203, 735) with the radius $R_{1}=9 \mathrm{kpc}$ and the effective semithickness $0.25 \mathrm{kpc}$. We took the model of the corona by Ninković, S., (1992, Astron. Nachr., 313, 83) where the parameters were established through the fitting.

From the rotation curve (using the points for $R / R_{0}>0.64$ ) we found the mass of the disk $4.6 \times 10^{10} \mathrm{M}_{(\cdot)}$, the mass of the corona (up to $68 \mathrm{kpc}$ ) $1.1 \times 10^{12} \mathrm{M}_{(:)}$and the mass of the core+bulge $1.9 \times 10^{10} \mathrm{M}_{(:)}$. We assume that the bulge is a spheroid of $0.6-0.8$ axial ratio. Using the rotation curve of Haud, U. (1979, Pis'ma $v$ Astron. $z h ., 5,124)$ for the central part of the Galaxy we found that the semimajor axis of the bulge is equal to $5 \mathrm{kpc}$, the central density $0.25 \mathrm{M}_{\circ} / \mathrm{pc}^{3}$, the density being constant up to $1.9 \mathrm{kpc}$ from the centre. For the density exponent of the outer part of the bulge we obtained the value -3 , for the bulge mass $14.6 \times 10^{9} \mathrm{M}_{(:)}$. 


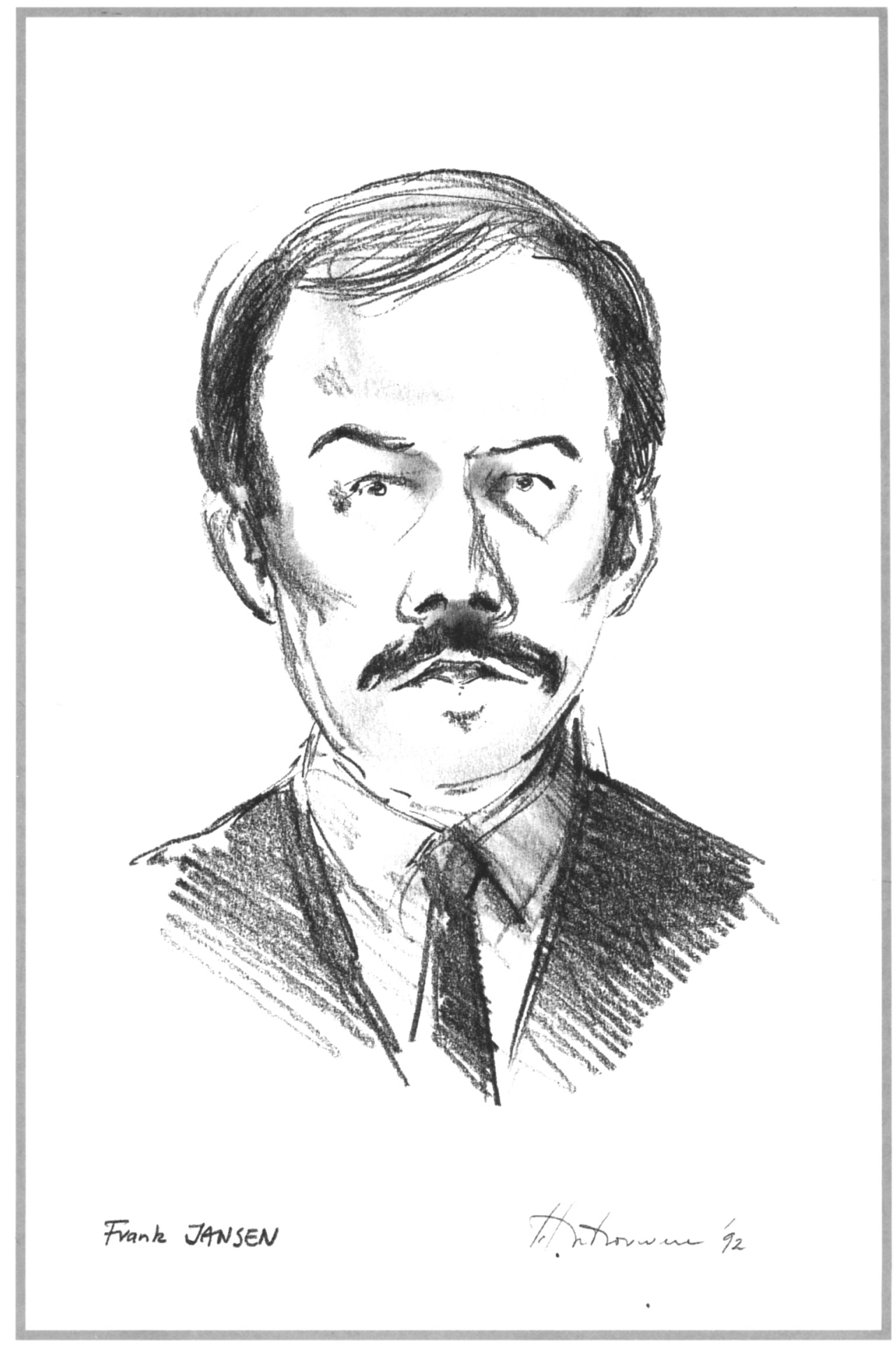

